\title{
Visual inspection with acetic acid for cervical cancer screening in a tertiary health care centre
}

\author{
Shaily Agarwal ${ }^{1}$, Renu Gupta ${ }^{1}$, Apurva Agarwal', Kiran Pandey ${ }^{1}$, \\ Neena Gupta ${ }^{1}$, Arti Katiyar ${ }^{1}$
}

\begin{abstract}
${ }^{1}$ Department of Obstetrics and Gynaecology, GSVM Medical College, Kanpur, UP, India
${ }^{2}$ Department of Anaesthesia, GSVM Medical College, Kanpur UP, India
\end{abstract}

Received: 06 January 2016

Accepted: 08 February 2016

\section{*Correspondence: \\ Dr. Shaily Agarwal, \\ E-mail: drspourush@gmail.com}

Copyright: () the author(s), publisher and licensee Medip Academy. This is an open-access article distributed under the terms of the Creative Commons Attribution Non-Commercial License, which permits unrestricted non-commercial use, distribution, and reproduction in any medium, provided the original work is properly cited.

\begin{abstract}
Background: Cervical cancer is one of the most prevalent malignant neoplasms among women in developing countries. Invasive cervical cancer is preceded by a long premalignant phase known as cervical intraepithelial neoplasia (CIN). The goal of cervical cancer screening is the detection and treatment of precancereous lesions before cancer develops. The objective of the study was to assess visual inspection with acetic acid (VIA) as a screening tool for use in a well-equipped health center, to evaluate VIA as an alternative or adjunct to the Papanicolaou (Pap) smear, and to determine if VIA can play a role in settings other than low resource ones.

Methods: This was a prospective study of 1520 asymptomatic women, carried out in 2014-2015. The study was performed at a tertiary care center equipped with the latest-generation technology and highly trained oncologists. The women underwent a complete clinical evaluation, including a Pap smear and VIA. Participants with any positive test were referred for colposcopy and biopsy.

Results: More women tested positive by VIA than on the Pap smear $(6.9 \%$ vs. $4.2 \%$; $\mathrm{P}=0.0001)$. There were 27 women with histologic cervical intraepithelial neoplasia grade 1 (CIN1); of these, 12 were detected by Pap and 15 by VIA $(\mathrm{P}=0.4)$. A diagnosis of CIN 2 or 3 (CIN2-3) was confirmed in a total of 11 cases; Pap detected 4 of the cases and VIA 9 of the cases $(\mathrm{P}=0.06)$. The positive predictive value for detection of CIN $2+$ was $8.5 \%$ for VIA and $6.2 \%$ for Pap $(\mathrm{P}=0.5)$. Most importantly, while only $2.8 \%$ of patients with a positive VIA were lost to follow-up before colposcopy that was true for $26.5 \%$ of the women with a positive Pap smear $(\mathrm{P}<0.0001)$.

Conclusions: VIA is useful for detection of precursor lesions of cervical cancer not only in low-resource settings but also in well-equipped health centers and cancer centers. In these non low-resource settings, VIA has a positive predictive value comparable to the conventional Pap smear, but it is more likely to achieve earlier diagnosis, followup, and treatment than cytology based screening.
\end{abstract}

Keywords: Cervix neoplasms, Acetic acid, Diagnostic techniques and procedure

\section{INTRODUCTION}

Cancer of the cervix uteri is the 4 th most common cancer among women worldwide, with an estimated 527,624 new cases and 265,653 deaths in 2012. The majority of cases are squamous cell carcinoma followed by adenocarcinomas. About 122,844 new cervical cancer cases are diagnosed annually in India (estimations for 2012). Cervical cancer ranks as the 2 nd cause of female cancer in India. ${ }^{1}$

Cervical cancer is one of the most prevalent malignant neoplasms among women in developing countries, affecting them mainly in the fifth to sixth decade of life. 
Invasive cervical cancer is preceded by a long premalignant phase known as cervical intraepithelial neoplasia (CIN). ${ }^{2}$ The goal of cervical cancer screening is the detection and treatment of precancer before cancer develops. ${ }^{3-5}$ To detect cervical intraepithelial neoplasia grades 2 or 3 (CIN 2-3), which are considered to be true precancerous lesions, we need a well implemented secondary prevention system that provides screening for all women at risk as well as treatment of detected abnormalities according to local policy. The Papanicolaou (Pap) smear has been shown to be highly effective in developed countries that have widespread screening programs. However, in India, as in most other developing countries, only a small proportion of women at risk are screened. In developing countries, because of the lack of trained cytotechnologists and cytology laboratories, there is often a long interval (1-3 months) between the Pap screenings and when the test result is available. $^{6}$ Additionally, only a small percentage of women with positive Pap smears have diagnostic evaluation and treatment, because of the lack of health centers that are able to treat preinvasive lesions. These problems with Pap smears have stimulated research on alternative tests, including visual inspection with acetic acid (VIA). VIA has demonstrated high sensitivity for detecting CIN and cervical cancer, but it is limited by low specificity. VIA is based on acetowhitening, with the CIN turning white when exposed to $5 \%$ acetic acid (vinegar). VIA has the advantage of requiring only low-technology equipments and the result is available within a couple of minutes. These characteristics make VIA a realistic alternative for low-resource settings. In India, cervical cancer screening based on the Pap smear is available in private and public health centers only in urban areas. However, there are several barriers to broad patient access to this screening. In addition, there is no nationwide quality control system to assure accurate screening. VIA has been only available in low-resource areas, The protocol described in this article was developed to evaluate whether VIA has a role as a screening test not only in first-level health centers in lowresource areas, but also in a tertiary health care center where Pap testing has been the norm. The Institute differs from low-resource settings in that it has the latestgeneration equipment, the physicians are highly trained specialists, and there is a well-established cytology laboratory that provides evaluation and diagnosis of cervical cytology.

\section{METHODS}

This study targeted sexually active women between 30 and 60 years old who had no history of CIN or cervical cancer (Although WHO recommends VIA screening for women in the age group between 30 and 49, but we screened women of age 30-60 so that these women between 50-60 age group who have never had a speculum examination at least get a speculum examination by a trained specialist). ${ }^{7}$ The women voluntarily visited the department of Obstetrics and gynaecology, G.S.V.M.
Medical College, Kanpur, between September 2014 and October 2015 for a general check-up and cervical cytology. We excluded women who were currently pregnant or who had a history of abnormal cytology, previous treatment for CIN or cancer, or obvious invasive cancer at the time of the clinical evaluation. Women with severe cervicitis were excluded until they had completed treatment. Women were included in the study after they were given information about the study, agreed to participate, and provided consent. Study subjects had a complete physical examination and pelvic evaluation done. The Pap smears sampling and VIA were performed by the same evaluator.

A speculum examination started with a direct visual evaluation of the cervix to identify cervicitis, leukorrhoea, polyps, ulcers, etc. A Pap smear sample was then taken using a conventional wooden Ayres spatula. The smear was fixed with ethanol for 30 minutes. VIA involved gentle application of $5 \%$ acetic acid using a small piece of cotton for one minute. After 1-2 minutes a naked-eye evaluation was performed under 100-watt illumination. The evaluator filled out a form with general information on each woman, clinical findings at pelvic evaluation, results of the VIA, and results of colposcopy when indicated. The results of the Pap smear were reported according to the Bethesda System. ${ }^{8} \mathrm{We}$ considered as positive any cytology diagnosis that included atypical squamous cells of undetermined significance (ASCUS), low-grade intraepithelial lesion (LSIL), high-grade intraepithelial lesion (HSIL), or invasive cancer. VIA was considered positive when an acetowhite lesion was observed within the transformation zone. If an acetowhite lesion was detected, the participant was immediately informed, and a colposcopy was done on the same day. Women with a negative VIA were given an appointment within a week to receive their Pap results and to have colposcopy if the Pap was positive. The time interval between the first evaluation, where the Pap smear and VIA were performed, and colposcopy was one week in women with a positive Pap smear. Colposcopy was performed. A directed biopsy was taken and fixed in $20 \%$ buffered formalin only if an acetowhite lesion was detected; women without acetowhite lesions during the colposcopic evaluation were considered as normal and did not have a biopsy. Pap smears were screened and reviewed by a pathologist. Biopsies were evaluated by pathologists blinded to the VIA results. For the statistical analysis, we considered as cases women with a final histology of CIN 2 or greater. Women with abnormal cytology or VIA but without colposcopy and biopsy were considered as lost to follow-up. They were excluded from the statistical analysis because they did not have a biopsy, and therefore, their final status is unknown. Observed data were analysed using spss software by chi square test.

\section{RESULTS}

We evaluated 1520 women who fulfilled the eligibility criteria and provided informed consent. Their mean age 
was 38.6 years. In terms of parity, $13.8 \%$ of the women were nulliparous, $45.6 \%$ had had one or two live births, $28.6 \%$ had had three or four, and $12 \%$ had had five or more. The mean age of onset of sexual life had been 19 years (median, 21 years; range, 13-41 years). The majority of the women $(95 \%)$ had had one sexual partner (Table 1).

Table 1: Demographic characterstics of the participants of the screening $(n=1520)$.

\begin{tabular}{|c|c|c|c|}
\hline & & No. & $\%$ \\
\hline \multirow{4}{*}{ Age (in years) } & $30-40$ & 696 & $45.78 \%$ \\
\hline & $41-50$ & 456 & $30.0 \%$ \\
\hline & $51-60$ & 368 & $24.20 \%$ \\
\hline & total & 1520 & $100 \%$ \\
\hline \multirow{5}{*}{ Parity } & Nulliparous & 210 & $13.21 \%$ \\
\hline & P1-P2 & 694 & $45.65 \%$ \\
\hline & P3-P4 & 436 & $28.68 \%$ \\
\hline & $\mathrm{P} \geq 5$ & 180 & $11.84 \%$ \\
\hline & Total & 1520 & $100 \%$ \\
\hline \multirow{3}{*}{ Habitat } & Rural & 836 & $55.0 \%$ \\
\hline & Urban & 684 & $45.0 \%$ \\
\hline & Total & 1520 & $100 \%$ \\
\hline \multirow{3}{*}{ Polygamy } & No & 1444 & $95.0 \%$ \\
\hline & Yes & 76 & $05.0 \%$ \\
\hline & Total & 1520 & $100 \%$ \\
\hline \multirow{7}{*}{$\begin{array}{l}\text { Age at first } \\
\text { sexual } \\
\text { intercourse }\end{array}$} & $<15$ years & 202 & $13.28 \%$ \\
\hline & 16-20years & 802 & $52.76 \%$ \\
\hline & $21-25$ years & 160 & $10.52 \%$ \\
\hline & $26-30$ years & 129 & $08.48 \%$ \\
\hline & $31-35$ years & 147 & $09.68 \%$ \\
\hline & $>35$ years & 80 & $05.26 \%$ \\
\hline & Total & 1520 & $100 \%$ \\
\hline
\end{tabular}

With regard to cytology history, $84 \%$ of the women had never had a Pap smear; only $4 \%$ had had one within the preceding year, $3 \%$ within one to three years, $5 \%$ within four to five years, and $4 \%$ more than five years before the study (Table 2).

Table 2: Distribution of study population according to cytology history $(n=1520)$.

\begin{tabular}{|lll|}
\hline & No. & $\%$ \\
\hline Never had cytology & 1276 & $84 \%$ \\
\hline$<1$ year & 60 & $04 \%$ \\
\hline 1-3 year & 48 & $03 \%$ \\
\hline 4-5 year & 76 & $05 \%$ \\
\hline$>5$ year & 60 & $04 \%$ \\
\hline
\end{tabular}

During the clinical evaluation (before the application of $5 \%$ acetic acid) 782 women $(51.4 \%)$ were observed as having a normal cervix, $554(36.4 \%)$ had cervicitis, $182(11.9 \%)$ had ectopy, and 2 women had leukoplakia. Of the 1520 women, 105 of them $(6.9 \%)$ had a positive VIA, and $64(4.2 \%)$ had a positive Pap smear $(\mathrm{P}=0.0001)$ (Table 3).

It is important to mention that 9 women $(0.6 \%)$ had a positive result on both tests; that is, 96 women $(6.3 \%)$ were only VIA positive, and 55 women $(3.6 \%)$ were only Pap-positive. Of the 105 women who had a positive VIA, 3 of them $(2.8 \%)$ did not return for colposcopy and biopsy. In contrast, of the 64 women with a positive Pap smear, 17 of them $(26.5 \%)$ did not return for colposcopy and biopsy $(\mathrm{P}<0.0001)$ (Table 4). These patients were lost despite efforts to contact them with phone calls to them and/or their relatives.

Table 3: Screening for cervical cancer done with visual inspection with acetic acid (VIA) and with conventional Pap smears.

\begin{tabular}{|llllllll|}
\hline \multirow{2}{*}{ Total $\mathrm{n}=1520$} & \multicolumn{2}{c}{ VIA positive } & \multicolumn{2}{c|}{ Pap smear positive } & \multicolumn{2}{c|}{ P value } & \multicolumn{2}{c|}{ VIA \& pap positive } \\
\cline { 2 - 8 } & 105 & 6.9 & 64 & 4.2 & 0.0001 & 9 & No. \\
\hline CIN 1 $(\mathrm{n}=27)$ & 15 & 55.55 & 12 & 44.44 & 0.4 & 1 & $3.7 \%$ \\
\hline CIN 2-3 $(\mathrm{n}=11)$ & 9 & $81.81 \%$ & 4 & $36.36 \%$ & 0.06 & 2 & $18.18 \%$ \\
\hline CIN 1-3 $(\mathrm{n}=38)$ & 24 & $63.15 \%$ & 16 & $42.10 \%$ & 0.09 & 3 & $7.89 \%$ \\
\hline
\end{tabular}

$\mathrm{P}$ value is for McNemar's chi-squared test.

Seventy-seven women underwent a biopsy during the study. 5 had a result of normal, 26 had cervicitis, 8 had metaplasia, 27 had CIN 1, and 11 had CIN 2-3. Among these 11 patients with a biopsy result of CIN 2-3, VIA was positive in 9 of them $(81.8 \%)$, and cytology was positive in 4 of them $(36.3 \%)$. The positive predictive value (PPV) for detection of CIN 2 or worse was $6.2 \%$ for the Pap smear and $8.5 \%$ for the VIA $(\mathrm{P}=0.5)$ (Table 4).
Table 4: Comparison of PPV and lost in follow up between VIA and pap smear.

\begin{tabular}{|c|c|c|c|c|c|}
\hline & \multicolumn{2}{|c|}{ VIA N=105 } & \multicolumn{2}{|c|}{$\begin{array}{l}\text { Pap smear } \\
\mathrm{N}=64\end{array}$} & \multirow{2}{*}{$\begin{array}{l}P \\
\text { value }\end{array}$} \\
\hline & No. & $\%$ & No. & $\%$ & \\
\hline $\begin{array}{l}\text { Predictive value } \\
\text { of positive test } \\
\text { (for CIN2-3) }\end{array}$ & 9 & $8.5 \%$ & 4 & $6.2 \%$ & $\mathrm{P}=0.5$ \\
\hline $\begin{array}{l}\text { No. of patients } \\
\text { lost in follow up }\end{array}$ & 3 & $2.8 \%$ & 17 & $\begin{array}{l}26.5 \\
\%\end{array}$ & $\begin{array}{l}P=0.0 \\
001\end{array}$ \\
\hline
\end{tabular}




\section{DISCUSSION}

Close to $80 \%$ of invasive cancer cases occur in developing countries, where either there are no screening programs or the programs are poorly developed and inefficient. Most of these programs are based on Pap smears and try to mimic the good results obtained in developed countries. Unfortunately, their results have been suboptimal due to lower coverage of women at risk, no standardized quality control systems, and a lack of follow-up and treatment of positive cases.

For these reasons, in recent decades other alternatives have been explored, such as human papillomavirus (HPV) DNA testing and VIA. HPV DNA testing has shown very high sensitivity and is being recommended in high-resource countries. ${ }^{9}$ However, its current price and technology requirements make this option unrealistic for poor areas. ${ }^{10}$ until a low-cost, same-day HPV test and realistic strategies are developed. ${ }^{11}$ VIA has arisen as a promising alternative for developing countries because it is inexpensive and fast and requires a low level of training and no special equipment. Some previous reports have observed that VIA can reach similar or better results than the Pap smear in the detection of CIN, Further, it is important to mention that there had been no prior experience with using VIA in a high-resource setting in a developing country. In general, the sensitivity of VIA has been shown to be equal to or better than the Pap smear's, while its specificity has been lower. We planned this study to determine the potential of VIA to supplement or replace the Pap smear in screening in a tertiary care center. It is important to highlight that our positivity rate for VIA was less than that reported by other authors, but we should emphasize that in our study the evaluators were highly trained gynecologists with experience in colposcopy, while in other studies the evaluators were health workers with less training. ${ }^{12-13}$ This finding highlights the importance of training and experience for the performance of visual evaluations. These issues should be considered during the development of protocols in remote areas, where we must ensure adequate training and volume of evaluations to maintain the expertise of the health worker. Another important finding concerned the percentages of women with positive tests who were lost to follow up before colposcopy and treatment. The percentage was significantly lower in VIA-positive women $(2.3 \%)$ than in women with a positive Pap smear $(26.3 \%)(\mathrm{P}<0.0001)$. We believe that this happened because the VIA positive women knew their abnormal result immediately during the first visit, and they immediately received special counselling about that finding and the importance of returning in a week for colposcopy and biopsy. In contrast, to learn about their Pap smear result (either positive or negative), women had to return one week later for a second visit, which many of them did not do and so never received special counselling about the significance of any positive result. This percentage of participants with abnormal cytology lost before colposcopy might be considered a source of bias at the time of statistical analysis and comparison with VIA. However, this result does reflect the real problems faced with screening based on the Pap test in developing countries. ${ }^{6}$ The positive predictive value we report for VIA is lower than that found by Sankaranarayanan et al. ${ }^{14}$ This can most likely be explained by our institutional policies, which required us to diagnose and follow up women with any lesion (cytological or visual) suggesting CIN 1. However, the PPV was calculated considering as cases only women with CIN 2-3 histology. Sankaranarayanan et al. found a PPV for VIA of $17 \%$, but they considered as positive at VIA only those cases with a distinctive and clear acetowhite area, which is more likely to be related to CIN 2-3. Our results outline the potential benefits of using VIA at all levels of health care systems in developing countries. $^{15}$

VIA increases detection of premalignant lesions of the cervix and diminishes the probability of losing women before they are appropriately followed up and treated. We believe that VIA can be used as a screening tool in poor countries not only in rural areas and small health centers, but also in tertiary care centers, district hospitals, cancer institutes, and other health facilities with better resources. As VIA is a real time test, biopsy and treatment can be performed in the same sitting, thus decreasing the probability of losing the patient.

\section{CONCLUSIONS}

VIA is useful for detection of precursor lesions of cervical cancer not only in low-resource settings but also in well-equipped health centers and cancer centers. In these non low-resource settings, VIA has a positive predictive value comparable to the conventional Pap smear, but it is more likely to achieve earlier diagnosis, follow-up, and treatment than cytology based screening.

\section{Funding: No funding sources}

Conflict of interest: None declared

Ethical approval: The study was approved by the Institutional Ethics Committee

\section{REFERENCES}

1. Human Papillomavirus and Related Diseases Report INDIA Version posted on www.hpvcentre.net in March $20^{\text {th }} .2015$.

2. Schiffman M, Kjaer SK. Chapter 2: natural history of anogenital human papillomavirus infection and neoplasia. J Natl Cancer Inst Monogr. 2003;(31):149.

3. Mahmoobeh S, Diane S, Philip EC. Cervical cancer prevention:Cervical screening, science in evolution. Obstet Gynecol Clin N Am. 2007;34:739-60.

4. Sehgal A. Human papilloma virus (HPV) and screening strategies for cervical cancer. Indian journal of Med Res. 2009;130:234-40. 
5. Sangwa LG, Salaheddin M. Visual inspection as a cervical cancer screening method in a primary health care setting in Africa. International Journal of Cancer. 2006;119:1389-95.

6. Gage JC, Ferreccio C, Gonzales M, Arroyo R, Huivin M, Robles SC. Follow-up care of women with an abnormal cytology in a low resource setting. Cancer Detect Prev. 2003;27(6):466-713.

7. WHO Comprehensive cervical cancer prevention and control - a healthier future for girls andwomen. http://www.who.int/reproductivehealth/publications/ cancers/9789241505147/en/(accessed27 Mar2014)

8. Apgar BS, Zoschnik L, Wright TC. The 2001Bethesda System terminology.Am Fam Physician. 2003;68:1992-8.

9. Wright TC, Schiffman M, Solomon D, Cox JT, Garcia F, Goldie S. Interim guidance for the use of human papillomavirus DNA testing as an adjunct to cervical cytology for screening. Obstet Gynecol. 2004;103(2):304-9.

10. Cronje HS. Screening for cervical cancer in developing countries. Int $\mathbf{J}$ Gynaecol Obstet. 2004;84(2):101-8.

11. Jeronimo J, Castle PE, Herrero R, Burk RD, Schiffman M. HPV testing and visual inspection for cervical cancer screening in resourcepoor regions. Int J Gynaecol Obstet. 2003;83(3):311-3.
12. Bhatla N, Mukhopadhyay A, Joshi S. Visual inspection for cervical cancer screening; evaluation by doctor versus paramedical worker. Indian Journal of Cancer. 2004;41:32-6.

13. Blumenthal PD, Lauterbach TM, Sellar JW, Sankarnarayanan R. Training for cervical cancer prevention program in low resource setting; focus on VIA and cryotherapy; International Journal of Gynaecology and Obstetrics. 2005;89:30-7.

14. Sankaranarayanan R, Budukh AM, Rajkumar R. Effective screening programmes for cervical cancer in low and middle income developing countries. Bull World Heal Organ. 2001;79:954-62.

15. Sankaranarayanan R, Esmy PO, Rajkumar R, Muwonge R, Swaminathan R, Shanthakumari S, et al. Effect of visual screening on cervical cancer incidence and mortality in Tamil Nadu, India: a cluster -randomized trial, Lancet. 2007; 370:398406.

Cite this article as: Agarwal S, Gupta R, Agarwal A, Pandey K, Gupta N, Katiyar A. Visual inspection with acetic acid for cervical cancer screening in a tertiary health care centre. Int J Reprod Contracept Obstet Gynecol 2016;5:752-6. 